\title{
Participation End Time
}

National Cancer Institute

\section{Source}

National Cancer Institute. Participation End Time. NCI Thesaurus. Code C162173.

The time of the end of a subject's participation in the study. 